\title{
OBSERVATORIO
}

\section{QUÉ ENTENDEMOS POR USUARIO COMO CENTRO DEL SERVICIO. ESTRATEGIA Y TÁCTICA EN MARKETING}

Nieves González-Fernández-Villavicencio

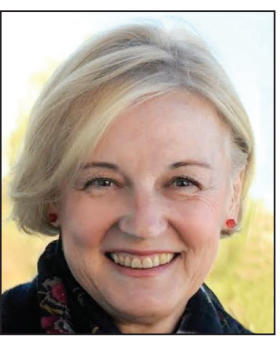

Nieves González Fernández-Villavicencio es responsable de la biblioteca de la Facultad de Económicas y Empresariales de la Universidad de Sevilla y profesora-responsable del Área de Biblioteconomía y Documentación de la Universidad Pablo de Olavide, también de Sevilla. Licenciada en geografía e historia por la Universidad de Sevilla y doctora en sociedad del conocimiento por la Universidad de Salamanca, sus líneas de investigación se han centrado en el marketing digital y los medios sociales, las competencias digitales y los servicios de referencia presencial y virtual. Es autora del blog Bibliotecarios 2020. http://orcid.org/0000-0001-8940-7429

Universidad de Sevilla, Biblioteca de la Facultad de Económicas y Empresariales Avda. Ramón y Cajal, 1. 41018 Sevilla, España nievesg@us.es

\section{Resumen}

Se expone la evolución del concepto de marketing para llegar a sus expresiones actuales como marketing de atracción, de contenidos y relacional, así como su especial implicación en el mundo de las bibliotecas y servicios de información. Se analiza la diferencia entre estrategia y táctica en marketing digital y se plantean las tendencias que los expertos ven más seguras a corto plazo, así como los retos que estas tendencias obligan a afrontar a las bibliotecas y organizaciones en general.

\section{Palabras clave}

Marketing digital; Marketing de servicios; Marketing de contenidos; Marketing relacional; Marketing de atracción; Medios sociales; Bibliotecas; Objetivo centrado en el usuario; Tendencias.

\section{Título: What does user focus mean? Marketing strategy and tactics}

\section{Abstract}

The development of a marketing concept is described, and current types are explained: inbound marketing, content marketing and relationship marketing, especially concerning libraries and information services. Marketing strategy and tactics are analyzed. Finally, current marketing trends that experts predict for the short term, and the new challenges related to these trends that libraries, and organizations in general, have to confront are discussed.

\section{Keywords}

Digital marketing; Inbound marketing; Relationship marketing; Content marketing; Social media; Libraries; Customer focus; Trends.

González-Fernández-Villavicencio, Nieves (2015). "Qué entendemos por usuario como centro del servicio. Estrategia y táctica en marketing". El profesional de la información, enero-febrero, v. 24, n. 1, pp. 5-13.

http://dx.doi.org/10.3145/epi.2015.ene.01

\section{Todo lo que significa marketing e ignoramos}

Marketing, mercadotecnia, mercadeo, estrategia comercial, publicidad, promoción, gestión del mercado, comunicación, branding..., son términos relacionados con el marketing, una ciencia que se basa en las relaciones entre las empresas/instituciones y sus clientes/usuarios así como sus intercambios de valor ${ }^{1}$ (Jiménez-Zarco et al., 2007).

Naturalmente el concepto de marketing ha ido evolucionando a lo largo del tiempo. Durante la primera mitad del s. XX el marketing se limitaba a tomar el producto y trasladarlo al mercado. Su rol consistía en colocar en el mercado lo que unos sesudos estrategas -léase también bibliotecarios- ideaban para los consumidores. De esta forma, los profesionales del marketing tomaban el producto en el momento final de su producción y a través de sus artes lo introducían a los compradores (Valls, 2014) -léase usuarios-.

Pero el marketing no es sólo una actividad de intercambio sino que utiliza técnicas que se basan en métodos, métricas y pautas de desarrollo para identificar necesidades y adelantarse a satisfacerlas. Durante la segunda mitad del s. XX el marketing pasa de mero soporte al management a disciplina 


\section{eli:Emprendedor.ec}

INICIO QUIÉNES SOMOS
EMPRENDIMIENTOS

CONTÁCTENOS

\section{Las nuevas reglas del marketing}

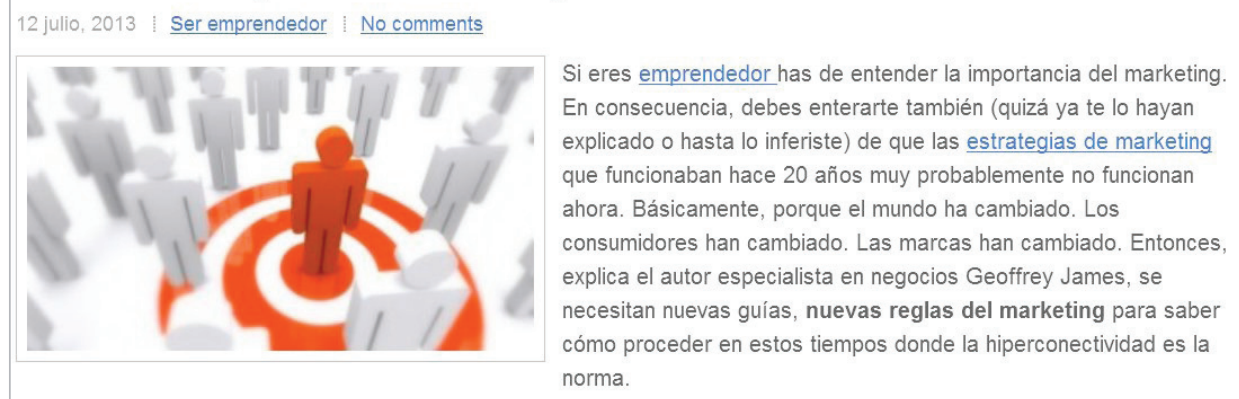

Figura 1. El emprendedor, una web con interesantes consejos: "...las estrategias de marketing que funcionaban hace 20 años muy probablemente no funcionan ahora".

http://www.elemprendedor.ec/nuevas-reglas-del-marketing

autónoma, a medida que se comienzan a aplicar conceptos como planificación, orientación al cliente y satisfacción de los usuarios. Un paso más allá lo vemos con Kotler (1999), el referente más destacado del marketing contemporáneo, que cambia el orden de los elementos y sitúa al consumidor en el origen de todos los procesos de gestión: no se trata de orientar el mercado hacia el cliente, de ponerse en su lugar, sino que el cliente es el origen de la gestión empresarial. Es el usuario el que dicta el producto o servicio que desea, quien decide el precio que está dispuesto a pagar e indica el canal a través del cual quiere recibirlo.

\section{Las bibliotecas deberían medir su grado} de orientación al mercado y cuáles son las aptitudes de sus líderes para hacer un buen marketing

En este enfoque la empresa captura el conocimiento del consumidor hacia su gestión interna. Si antes el marketing era investigación de mercado, publicidad y venta, ahora consiste en captar las percepciones, las preferencias y el entorno de la toma de decisiones de los clientes (figura 1). Esa mayor participación del consumidor está transformando las relaciones de marketing. Escuchar al consumidor e implicarle en la construcción de los productos lleva al éxito, y hacer lo contrario lleva al fracaso. El denominado marketing estratégico es de esta forma superior al táctico -el segundo se subordina al primero a partir de las demandas de los consumidores-.

Sin embargo la realidad es que los departamentos de marketing de las organizaciones -no lo olvidemos, incluidas las bibliotecas-, aún siguen anclados a la era pre-Kotler ${ }^{2}$ ya que para acomodarse a esta perspectiva las empresas requieren de dos visiones (Fava, 2013). La primera, una actitud emprendedora de constante adaptación al cambio aplicable también a sus jerarquías, estrategias y procesos. La segunda, la interconexión de las empresas en una red global, desarrollando una mentalidad colectiva de servicio a esta causa, de alianza con todos sus stakeholders (personas interesadas).
FORO ELEMPRENDEDOR

El cliente es importante, pero hay también otros grupos de interés a los que hay que servir. La clave está en ser capaces de establecer un diálogo continuo y mantenido en el tiempo. Indiscutiblemente, hoy día los medios sociales son los canales de comunicación con más posibilidades, y el entorno digital y móvil facilita, potencia y transforma todo este proceso. Ya Kotler se preguntaba para qué servían los estudios de mercado cuando resultaba mucho más sencillo conocer lo que piden los consumidores a través de una buena alianza con ellos.

\section{Apellidos del marketing: de atracción, de contenidos y relacional}

Meerman (2011) indica en su mediático libro que existen nuevas reglas de marketing que superan la simple publicidad ya que la audiencia ha cambiado y ahora exige transparencia y autenticidad y quiere ser parte de la marca. No quiere ser interrumpida sino recibir los contenidos en el momento en el que los necesita. Con esta visión de orientación al cliente lo que está en juego es captar lo que éste desea, elemento clave del denominado marketing de atracción. Es el marketing centrado en personas, que Brian Halligan definió como inbound marketing hace ya casi 10 años. Al final, escuchar al cliente, emocionarle y enamorarlo es la base para conseguir un satisfactorio desarrollo del proceso de compra. Catuxa Seoane lleva años contando cómo aplican este marketing en las bibliotecas municipales de $A$ Coruña (Seoane-García, 2012).

\section{Es el usuario quien dicta el producto o servicio que desea, el precio que está dispuesto a pagar y el canal a través del cual quiere recibirlo}

Para diferenciarse de los competidores que también están buscando la forma de conocer qué quiere el cliente, hay que ofrecerle y enseñarle nuevas oportunidades o iniciativas y vincularse a metas que les motiven. El marketing por una parte aprende del consumidor pero por otra le va enseñando con contenidos relevantes, entretenidos y oportunos que le aporten algo. Es aquí donde aparece en escena el marketing de contenidos, el alimento indispensable de los medios sociales. Los auténticos profesionales del marketing digital, los content curators y community managers, gestores de contenidos y comunidades en palabras de MerloVega (2014), usan su inteligencia y creatividad para crear conexiones con su público -claramente definido- mediante un contenido valioso, relevante y coherente, que lo atraiga, retenga y en última instancia impulse una acción rentable 
del cliente ${ }^{3}$. Una acción es rentable cuando convierte, o sea, cuando el cliente compra, cosa que en el negocio de la biblioteca podemos traducir por un mayor uso de sus productos y servicios, y en general por un mayor impacto de lo que realizan los bibliotecarios.

El proceso mediante el cual se identifican los clientes potenciales para establecer relaciones con ellos es lo que se denomina marketing relacional, algo que sólo se consigue mediante su profundo conocimiento y un trato personalizado, con estrategias diferenciadas por cada canal de comunicación utilizado, cada target (público objetivo) y cada objetivo. Lo que se pretende es mantener y acrecentar esas relaciones para fidelizar a los clientes y convertirlos en prescriptores de nuestro producto o servicio, alcanzar una importante cuota de clientes comprometidos con nuestra marca y convertir a los clientes esporádicos en fieles. Esto sólo se consigue generando una gran experiencia de marca y una elevada satisfacción en su relación con nuestra empresa/biblioteca y en cada una de las fases de esa relación.

En el entorno de las bibliotecas siempre se han tenido grupos de usuarios fieles cuyas necesidades se han visto sobradamente cumplidas con los servicios bibliotecarios. Sin embargo el reto ahora es no sólo no perder esos usuarios cuyas necesidades en relación a la biblioteca están cambiando, sino alcanzar una nueva audiencia, llegar a otros colectivos que no son usuarios, a los que hay que recordarles la existencia de nuestra marca y construir relaciones con ellos cuando se plantean necesidades en las que la biblioteca puede ayudarles.

Para el experto en marketing Geoffrey James ${ }^{4}$ la diferencia entre el marketing tradicional y el relacional se establece en base al siguiente cuadro:

\begin{tabular}{|l|l|}
\hline \multicolumn{1}{|c|}{ Tradicional } & \multicolumn{1}{c|}{ Relacional } \\
\hline $\begin{array}{l}\text { Crea un producto que } \\
\text { coincide con los intereses de } \\
\text { compra de un alto número de } \\
\text { personas o posibles compra- } \\
\text { dores. }\end{array}$ & $\begin{array}{l}\text { Crea un producto que apela a } \\
\text { los intereses de compra de un } \\
\text { consumidor específico, por lo que } \\
\text { es necesario que la audiencia esté } \\
\text { claramente definida y segmentada. }\end{array}$ \\
\hline $\begin{array}{l}\text { Emite el mensaje del producto } \\
\text { ante una audiencia lo más am- } \\
\text { plia posible. Apunta a todos } \\
\text { los posibles compradores que } \\
\text { puede. }\end{array}$ & $\begin{array}{l}\text { Dirige el mensaje del producto a } \\
\text { esa audiencia inicial, con el objeti- } \\
\text { vo de convertirlos en fans a través } \\
\text { de un contenido valioso, relevante } \\
\text { y coherente para atraer y retener a } \\
\text { esa audiencia. }\end{array}$ \\
\hline $\begin{array}{l}\text { Crea una marca de producto } \\
\text { reconocible, que puede luego } \\
\text { extenderse a otras categorías } \\
\text { de producto. }\end{array}$ & $\begin{array}{l}\text { Deja que las preferencias de esos } \\
\text { fans definan la marca y el futuro de } \\
\text { las ofertas. }\end{array}$ \\
\hline
\end{tabular}

Internet ha fragmentado las audiencias en grupos pequeños de consumidores con intereses y características específicas. Por esta razón los mensajes deben ser muy personalizados para despertar interés y evitar el abandono. En la actualidad hay tantos productos, servicios y marcas ofertándose en todas partes, que los consumidores simplemente no las perciben, por lo que hay que diferenciarse de la competencia.

\section{Una acción es rentable cuando convier- te. En el negocio de la biblioteca pode- mos traducirlo por un mayor uso de sus productos, servicios e impacto de sus bibliotecarios}

Algunos autores hablan de la evolución del marketing hacia el 3.0 o semántico. De esta forma hemos pasado de un marketing centrado en el producto (marketing 1.0) a otro centrado en el usuario, buscando su participación (marketing 2.0), y así llegamos al marketing centrado en valores y búsqueda de significados (marketing 3.0). En este último sentido, las empresas no sólo buscan generar beneficios sino un comportamiento responsable hacia la humanidad y el planeta, en un marketing social, colaborativo, cultural y espiritual (Kotler, 2011). El marketing interactivo y social utiliza los principios del marketing y las técnicas de influencia del público objetivo, el target, para aceptar voluntariamente, modificar o abandonar una conducta si con ello presta un mejor servicio a los individuos, los grupos o la sociedad. Junto a ello hay que incorporar el concepto de marketing verde entendido como la manera de compatibilizar las necesidades de los clientes con la protección del entorno, los productos ecológicos y sanos, aunque ello implique costes superiores. Se trata de incluir en 


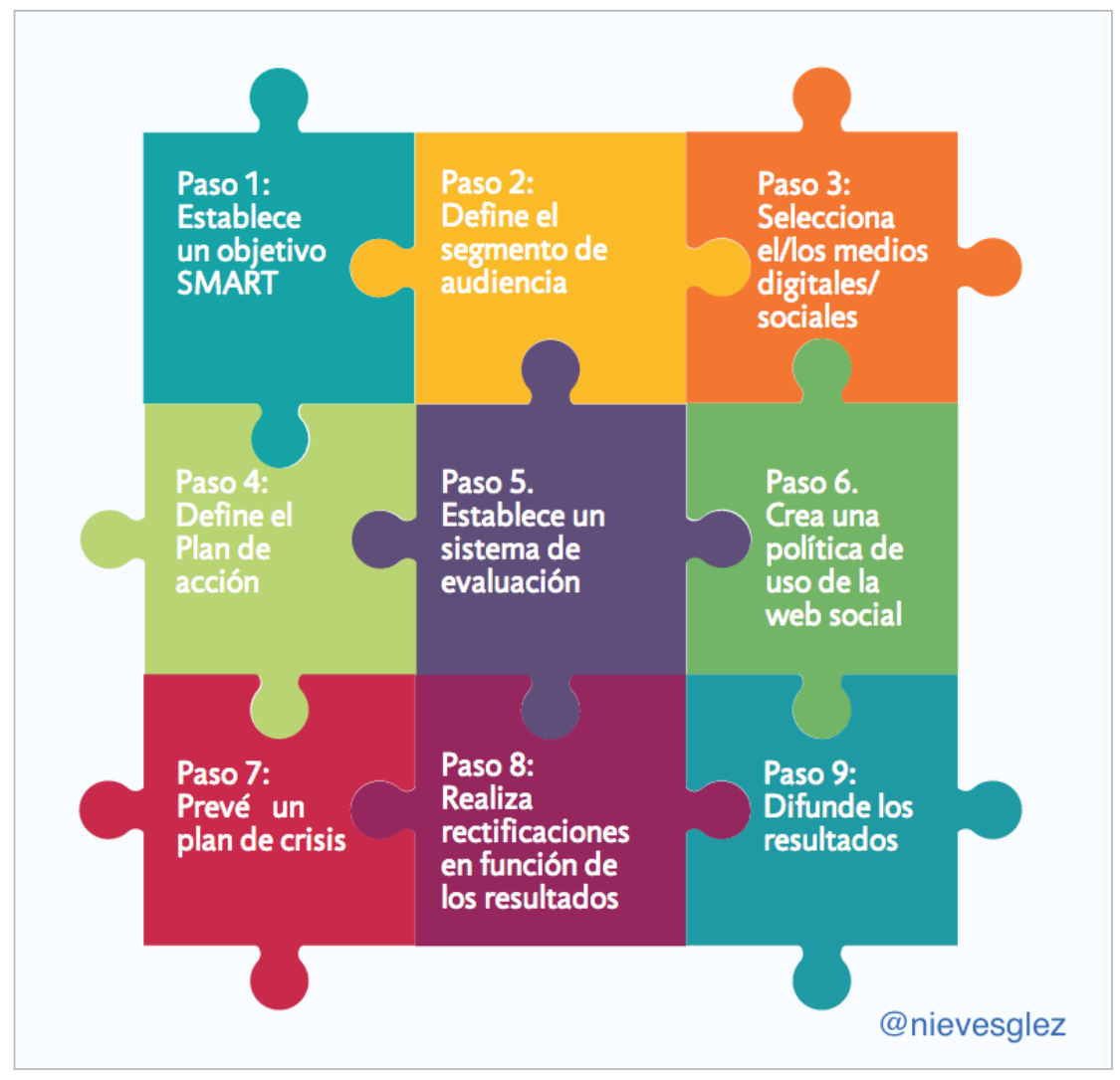

Figura 3. Componentes de un plan de marketing

https://magic.piktochart.com/output/2322885-marketing-library

el cometido de buscar la satisfacción del cliente, la función social y medioambiental de la empresa.

\section{Hacen falta sistemas de métricas comu- nes para medir el impacto de las accio- nes online, que funcionen para todas las organizaciones}

\section{El marketing tiene una estrategia y una táctica}

Tradicionalmente en marketing se habla de estrategia y táctica. En términos simples, una estrategia es un plan para alcanzar una meta específica, mientras que una táctica consiste en los medios concretos que se usan para alcanzar esa meta.

La estrategia del marketing requiere una planificación para alcanzar unos objetivos previamente definidos que tienen que ser SMART, utilizando las cinco reglas del acrónimo inglés: Specific, Measurable, Achievable, Realistic, Timely. Esta planificación incluye contabilizar los esfuerzos colectivos de los actores implicados, asignar recursos, temas de liderazgo, planificación temporal así como el análisis sobre la situación de la institución, de su mercado y de su competencia (figura 3). La táctica consiste en definir las acciones que se tienen que realizar para conseguir el objetivo estratégico, o sea, la forma en la que vamos a implementar la estrategia con las acciones que se van a llevar a cabo. Esto se resume en el marketing estratégico (pensar) y el operativo (actuar). La estrategia y la táctica son dos caras de una misma moneda, pero ambas se condicionan mutuamente, y ambas siempre deben estar documentadas, no ser únicamente verbales para tener más probabilidades de éxito ${ }^{5}$.

Los medios sociales se han constituido en los canales operativos más adecuados, y de hecho durante mucho tiempo se han asociado a las tácticas digitales recomendables para conseguir los objetivos estratégicos. Junto a los medios sociales se han situado los dispositivos y aplicaciones móviles, sin los cuales no habrían llegado a ser lo que son. Los canales digitales no sólo permiten sino que obligan a un modelo de relación bidireccional entre iguales. Como decimos, durante años el marketing digital se ha asociado a una perspectiva táctica, por ejemplo conseguir muchos fans en Facebook. Sin embargo la evolución y consolidación del entorno digital ha demostrado que la transformación digital es mayor y de carácter estratégico. Las instituciones que triunfan utilizan las nuevas tecnologías para transformar sus modelos de negocio y aprovechan las oportunidades para generar mejores experiencias fidelizadoras en sus clientes/lectores. Para el director de marketing David Berkowitz", "el acercamiento a la publicidad en los medios sociales no sólo puede ser un movimiento táctico sino completamente estratégico para las marcas y para la forma en la que éstas llegan al mercado. La colaboración que permiten con los consumidores hace que las marcas se construyan de forma diferente y logran, por otro lado, que se hagan mejores productos, más adecuados a lo que quiere el mercado".

\section{Toda acción o campaña de marketing debe hacerse pensando que tiene que funcionar en el móvil}

Tanto para establecer las estrategias como las tácticas hay que conocer muy bien cuáles son las necesidades de los usuarios. La tendencia es a centrarse más en las acciones online frente a las offline, pero todas deben estar integradas y sobre todo, lo más importante es conocer, llegar a averiguar, dónde quieren nuestros usuarios ser contactados, algo que no lo definen ni el mercado ni las tendencias más innovadoras. Debemos escuchar a nuestros usuarios, saber qué propuestas de las que les hacemos, valoran y aprecian más. No basta con ponerse en su lugar: es el usuario el que tiene que ser escuchado antes de establecer ninguna estrategia de marketing. En este contexto, uno de los principales factores de éxito es la segmentación de la audiencia. Las cuatro características que debe tener la segmentación de usuarios son:

1) tamaño, suficientemente amplio como para justificar la inversión de las acciones en marketing;

2) diferenciación clara de otros segmentos; 
3) rentabilidad, con un potencial que justifique la estrategia;

4) accesibilidad, que se pueda llegar a ellos y exista posibilidad de contacto.

Pero además el reto del marketing actual es que los responsables del mismo en las organizaciones tienen que hablar el lenguaje de los negocios, traduciendo sus esfuerzos e inversiones en términos de resultados, retorno y creación de valor (Villaseca-Morales, 2014).

\section{Qué cultura del marketing tienen las bibliotecas}

El marketing de productos y servicios de información es de gran interés para los profesionales de la información. Aunque han sido las organizaciones de gran consumo las que le han dado más peso al marketing, recientemente las empresas de servicios están aumentando ese interés (VillasecaMorales, 2014).

Deborah Lee (2013) describe en su columna sobre marketing de la revista Public services quarterly lo que éste puede hacer por la biblioteca, ya que marketing es establecer un canal de comunicación entre un producto o servicio y su público objetivo. El proceso del marketing, como hemos visto, no es vender un producto sino identificar las necesidades de los usuarios y definir la forma en la que el producto o servicio satisface esas necesidades; es investigar qué necesita el cliente para poder crear productos adecuados para él, suficientemente atractivos y ofrecidos cuando y donde se necesitan. Los bibliotecarios deben ser conscientes de las variadas necesidades de su audiencia para ofrecer los servicios que sean más útiles a cada segmento de la misma.

La mayoría de las bibliotecas españolas no tiene un plan estratégico y sólo asume el marketing desde un punto de vista táctico, a corto plazo

Las actividades de marketing constituyen el día a día de los bibliotecarios, tal como se expone en la primera obra de la colección Elsevier's learning trends series dedicada a Marketing the academic library, que recopila capítulos de varios libros. En esta obra se ofrecen recomendaciones para las bibliotecas universitarias enfocadas sobre todo a llevar a cabo iniciativas y acciones de marketing en el contexto de la universidad, con fines de visibilidad e influencia y tanto con medios online como tradicionales.

El debate sobre el grado de visibilidad de los bibliotecarios en sus organizaciones y en la sociedad, vuelve a surgir. ¿Realmente los stakeholders de los que depende la biblioteca están al tanto de lo que hacen sus bibliotecarios? ¿Es a ellos a los que los bibliotecarios informan de sus actividades? Y esos informes ¿constituyen sesudas y pesadas memorias anuales que se publican cuando ya no se necesitan para la toma de decisiones, o por el contrario son de fácil lectura, con gráficos, y de mayor frecuencia y actualización?

Podemos decir que, aunque exista una abundante bibliografía sobre marketing y servicios de información y ejemplos de buenas prácticas, la mayoría de los bibliotecarios adolecen de habilidades para el marketing, las relaciones públicas y la autopromoción (Elsevier, 2014). No es difícil encontrar hoy día bibliotecarios que utilizan el término "mercadeo" para despreciar acciones de marketing, con un valor peyorativo, ya que no ven con buenos ojos la idea de llevar a cabo técnicas de marketing que tengan connotaciones de "venta" o "negocio" para las bibliotecas (Polger; Okamoto, 2013). Sin embargo insistimos: para ser un buen bibliotecario hay que tener habilidades de networking, autopromoción y marketing, o peligra seriamente el futuro de la institución.

\section{A mayor segmentación, mayores proba- bilidades de conversión (usuarios poten- ciales convertidos en reales)}

Aunque en la actualidad las bibliotecas asumen como tarea propia la difusión de los servicios -véanse como ejemplo los paneles de LibraryAware (Ebsco $)^{7}$ en Pinterest para la promoción de los servicios bibliotecarios-, no se aplica el marketing desde un punto de vista estratégico sino táctico y son escasos los planes en ejecución que hagan suponer la consolidación de una auténtica cultura entre las bibliotecas españolas.

En cambio, investigaciones sobre la existencia de esta cultura en bibliotecas de otros países (Singh, 2009) ponen de manifiesto que se trata de un conjunto de creencias y conocimientos sobre marketing y la implementación de una serie de actividades que convierten la cultura en una práctica orientada al mercado y llevan a que la biblioteca ofrezca un servicio de mayor calidad, que se corresponde con una alta satisfacción de sus usuarios. Como parte imprescindible hay que dedicarle el tiempo suficiente a las relaciones con los influyentes y con los que toman las decisiones. Hay que pensar de forma estratégica. No nos "van a dar el sitio" que nos merecemos ni el reconocimiento justo, ni siquiera nos van a mantener en nuestro puesto de trabajo porque seamos buenos, que sin duda lo somos, sino porque les estamos resolviendo sus problemas, les estamos siendo útiles, les facilitamos las cosas. Vivimos en la época de la sobreabundancia de información, no podemos seguir pensando lo que se están perdiendo al no usar los recursos y servicios que la biblioteca les ofrece, sino en qué medida podemos ayudarles. Ése es el reto.

¿Por qué unas bibliotecas están más orientadas al marketing que otras? Por la propia cultura de la organización. No se trata de la actitud del personal de las organizaciones sino de los principios que subyacen bajo los comportamientos y las actitudes compartidas por los miembros. Las bibliotecas deberían medir su actual nivel de orientación al mercado y cuáles son las características de sus líderes en relación con el marketing así como las actitudes del personal, la estructura de la biblioteca, los procedimientos y políticas y su sistema de funcionamiento interno (Singh, 2009).

\section{Tendencias del marketing de productos y servicios}

A finales de año la web se llena de previsiones y tendencias. Veamos algunas de las más destacadas ${ }^{8}$ (figura 4) para el 
Wheelhouse

\section{Mredictionsfor2015}

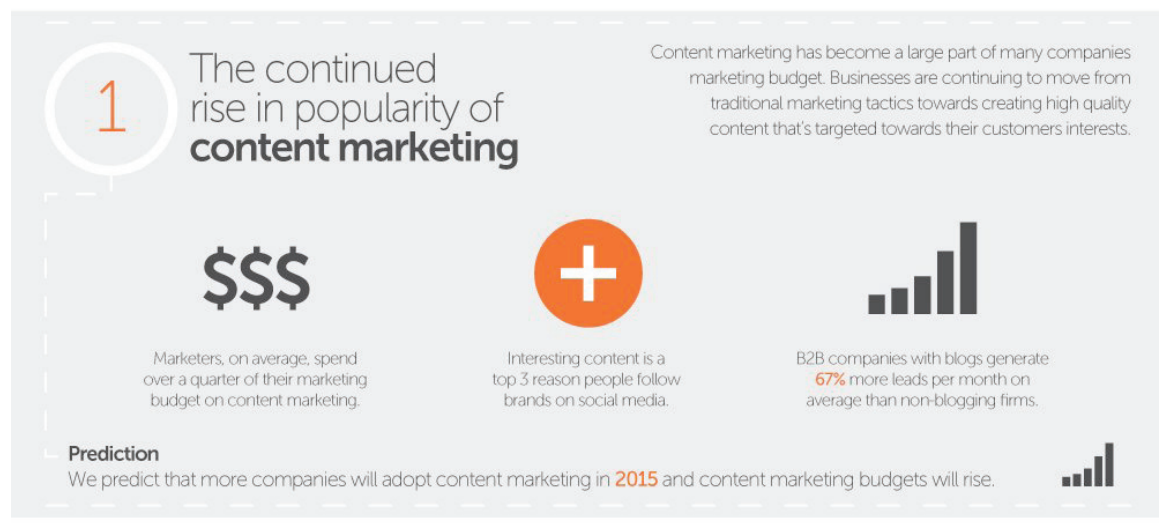

Figura 4. La infografía de Wheelhouse recoge 10 tendencias en marketing online para 2015. En la ilustración se muestra la primera de ellas.

http://i.imgur.com/M8p4tlk.png

marketing en 2015, todas de estratégica aplicación en bibliotecas y servicios de información.

\section{Marketing móvil}

En primer lugar y de forma redundante aparece el marketing móvil y la tendencia hacia el aumento de servicios a través de estos dispositivos, lo que conllevará a una mayor inversión tanto en medios sociales como en soluciones en movilidad con fines de marketing. Se estima que en 2019 habrá más del doble de smartphones, PCs, tablets, coches conectados y dispositivos wearable ${ }^{9}$. Este dato es especialmente interesante en el entorno español, el país con mayor penetración mundial en smartphones: el $83 \%$ de los móviles ${ }^{10}$. Sin embargo, no todas las organizaciones disponen de una estrategia multicanal integrada, ni están trabajando en ello. Y mucho menos si hablamos de las bibliotecas, que continúan enfocándolo desde una perspectiva táctica.

Las estrategias y los planes de marketing de 2015 serán distintos de los años anteriores debido en primer lugar a las tecnologías móviles. Las webs adaptativas (adaptables al tamaño de la pantalla) serán una constante frente a las apps, como ha sido hasta ahora. De hecho, el marketing debería seguir una estrategia de mobile first (primero móvil), de tal manera que cualquier acción o campaña debe hacerse pensando que tiene que funcionar en el móvil.

\section{Uso multicanal, integración, autoservicio e inmedia- tez}

Cuatro características en las que coinciden las previsiones para el marketing del 2015 son el uso multicanal y la integración, el autoservicio y la inmediatez. Veremos cómo se empiezan a integrar distintos medios entre sí, sobre todo con medios sociales: transmisiones de vídeo en streaming desde las páginas de Facebook, streams de Twitter dentro de sitios web para seguir la cobertura de eventos, pantallas interactivas mostrando streams de Pinterest y Twitter o plataformas y botones de compra directa en redes sociales. Todo esto deberá ser adaptativo y pensado para que pueda ser consumido o visto en los distintos dispositivos móviles. El autoservicio implica no imponer cuándo, dónde y de qué modo se establecen las relaciones con las organizaciones, y está demostrado que los clientes que reciben de la marca una respuesta rápida son tres veces más propensos a volver a usar y recomendar la marca ${ }^{11}$. No estaría de más que las bibliotecas revisaran sus tiempos de respuesta al usuario en los servicios de referencia virtual, en las cuentas de Twitter o Facebook y demás medios digitales.

\section{Marketing en tiempo real}

Otra de las constantes en la lista de predicciones es el marketing en tiempo real, que se relaciona con la personalización de los contenidos cuando se responde a los consumidores de manera más objetiva y segmentada. El mundo de la llamada internet de las cosas nos permitirá reaccionar en tiempo real de un modo hasta ahora inimaginable. No se trata sólo de que llegue a la audiencia específica para la que fue creado, sino que llegue en el momento oportuno, en el que se necesita. De esta forma ganamos todos, por un lado los servicios de información, ya que se conoce el servicio o producto cuando se necesita y de esa forma hay más probabilidad de que se utilice, y por otro lado el usuario, al que no se le hace perder el tiempo.

Para ello en primer lugar tenemos que empezar con datos procedentes básicamente de los medios sociales que deben ser analizados, filtrados, distribuidos. Pero también podemos obtener información a través de los comportamientos de los usuarios en las bases de datos, los catálogos de las bibliotecas, las webs, los repositorios, etc.

En segundo lugar hay que añadir esa información que aportan los datos a la experiencia de los profesionales. No dejarlo sólo en datos. Sabiendo lo que se demanda, y lo que se demanda de forma recurrente, podemos predecir lo que va a suceder, para lo cual hay que planificar, adelantarse y modificar en la medida que cambien las necesidades, con la mayor flexibilidad y adaptación posible.

Ponemos como ejemplo la alianza de IBM y Twitter para transformar la toma de decisiones en las empresas ${ }^{12}$. La integración de los datos que ofrece Twitter, auténtico sintetizador de tendencias, con los servicios de analítica en la nube de $I B M$, cambiará la forma en la que se toman las decisiones ya que se van a identificar las oportunidades que conecten 
mejor con los clientes, dirigidas a mejorar la relación realizando un mapa de sentimientos y comportamientos de los usuarios, y al mismo tiempo se podrán predecir tendencias basadas en patrones en tiempo real. Quizá las bibliotecas podremos tener un mapa en tiempo real de las necesidades, comportamientos y sentimientos de nuestros usuarios, para ofrecerles productos y servicios a su medida en cada momento...

Para ser un buen bibliotecario hay que tener habilidades de networking, autopromoción y marketing

\section{Retos que plantean las tendencias}

Las anteriores tendencias digitales presentan sin duda retos a las empresas y organizaciones que Leeflang (2014) identificó y cuantificó a partir de entrevistas a 777 ejecutivos e investigadores del área del marketing de los EUA, Europa y Asia. Veamos esos retos.

Revolución digital y cambio del modelo de negocio. Las herramientas y tecnologías digitales amenazan los modelos tradicionales de negocio, de tal forma que se hace necesaria una estrategia digital desde la perspectiva del cliente; la experiencia del usuario (UX) adquiere en la economía digital un mayor peso. Las tácticas en medios sociales y móviles deben transformarse en una verdadera estrategia transformadora de marketing móvil y social.

Conocimiento del usuario/cliente. Para diferenciarse de la competencia es imprescindible poder segmentar y personalizar la experiencia de marca de nuestros clientes, empleados y colaboradores en función de lo que conocemos de ellos. Incluso por el simple modo de navegar en una web o una aplicación móvil, podemos deducir sus intereses y ofrecerles los contenidos más relevantes. Recordemos que la personalización será la clave del éxito en las acciones de marketing online, ya que el $74 \%$ de los clientes no puede evitar su frustración ante un contenido irrelevante ${ }^{13}$. A mayor segmentación, mayores probabilidades de conversión -usuarios potenciales convertidos en reales-. Sirvan como ejemplo los recientes lanzamientos de servicios de microlocalización que van a permitir que las bibliotecas envíen mensajes segmentados por área a través de Bluetooth y tecnología Beacon, tanto a dispositivos Android -el más extendido en el mercado- como iOS. Además, en este entorno digital los big data son la norma: procesar una gran cantidad de datos que ofrecen información sobre el comportamiento de las personas. Esto implica capturar los datos, gestionarlos, almacenarlos, recuperarlos, compartirlos, transferirlos y sobre todo, analizarlos y visualizarlos. Para el caso de los servicios de información, significa seguir al usuario en su proceso de adquisición de información, conocer cómo reacciona ante la oferta cada vez más segmentada de nuestros productos y servicios, cómo se comporta en los espacios web destinados a su uso. Sobre este aspecto de los big data o social big data puede verse el monográfico de noviembrediciembre de 2014 de El profesional de la información, concretamente los artículos de Serrano-Cobos (2014) y Martínez-Martínez y Lara-Navarra (2014).
Ruptura para avanzar. Aquí se plantea la disyuntiva procesamiento de datos frente a creatividad. La sobreabundancia de datos puede ahogar la creatividad y la innovación disruptiva. Aunque la creatividad y la innovación se puedan ver reducidas en un entorno de decisiones basadas en datos, no implica necesariamente que no haya innovación. Es el grado de innovación el que se verá afectado, aunque los big data también se consideran fuente de innovación, incluso pueden suponer un aumento de la eficiencia y la productividad.

Medios sociales. El reto que se plantea es la existencia de un usuario comprometido frente al usuario enfurecido. Los medios sociales se pueden usar para diversos fines, como forma de atención al cliente, para gestionar la reputación de la marca y monitorizar lo que se dice de ella, como forma de hacer oír la voz de la marca, como forma de adquirir clientes, para las relaciones públicas, analizar la competencia, crear comunidad en torno a la marca, solucionar problemas, visibilidad online y valor de la marca. Gestionar la salud y la reputación de la marca es un reto del entorno de marketing en el que los medios sociales juegan un importante papel. Todos quieren tener una estrategia en los medios sociales y aquellas organizaciones que cuenten con una estrategia digital bien definida sabrán sacar partido de la innovación digital y serán líderes en sus segmentos de mercado. Aquellas que no presten atención a estos aspectos probablemente verán amenazadas sus fuentes actuales de facturación (Tiago; Veríssimo, 2014). Sin embargo, antes de usar estos medios para oír la voz de los usuarios, hay que hacer un estudio de la percepción de la marca. Empresas que tengan opositores activos en los medios sociales, tienen el riesgo de no tener éxito en sus campañas en estos medios (caso McDonald's ${ }^{14}$ ).

Oportunidad online. Aquí nos enfrentamos a la cuestión de la segmentación digital, los jóvenes frente al resto de la audiencia. Con frecuencia el marketing digital se dirige al segmento de los jóvenes y descuida los prometedores grupos de otras edades. Si en los comienzos del mundo digital los usuarios de estos medios eran los jóvenes, actualmente la franja de edad que crece con más energía es la de los mayores, por lo que habrá que combinar ambas estrategias, la online y la tradicional.

Los recursos han de destinarse a las oportunidades, no a los problemas (Drucker)

Interacciones automatizadas. La automatización del servicio y la presión por migrar las interacciones con los clientes al entorno online puede causar insatisfacción en el cliente y destrucción del valor de la marca. Hay que realizar un estudio para ver qué es lo que se va a migrar y a qué colectivo le va a afectar. Hay migraciones que han sido muy bien acogidas como la banca online o la administración electrónica, siempre que sea fácil utilizarlas, funcionen bien y no nos compliquen la vida.

Métricas. Evaluar la efectividad del marketing digital es difícil ya que las métricas tradicionales no sirven. No se pueden trasladar los sistemas de medida de un entorno presencial al digital, como tampoco se pueden utilizar únicamente mé- 
tricas que midan el alcance en los medios sociales, las vanity metrics. Hacen falta métricas de impacto, actionable metrics, de sentimiento, cuantitativas y cualitativas, de retorno y de conversión. Hacen falta sistemas de métricas comunes que midan el impacto de las acciones online, y que funcionen para todas las organizaciones.

Gap de talento analítico. El marketing y los departamentos relacionados de las organizaciones se están enfrentando a un significativo gap de talento analítico. La sociedad de la información se caracteriza por la gran cantidad de datos que se reciben y a los que se tiene acceso cada día, lo que hace imprescindible la intervención de un profesional que los filtre, ordene, priorice y facilite a otras personas para que hagan uso de la información procesada. Las empresas que analizan sistemáticamente los datos que pueden conseguir sobre sus usuarios son las más competitivas y las que destacan frente a sus competidores. Volvemos a los big data y el análisis de datos que se consideran año tras años como las tendencias más seguras en los informes Horizon ${ }^{15}$. Se buscan personas con talento analítico: según datos del McKinsey Global Institute ${ }^{16}$ habrá una demanda de entre 140.000 y 190.000 analistas de datos en EUA en 2018, con conocimientos de estadística, matemáticas y econometría. La formación se ha centrado en la atención directa al cliente o a los nuevos canales, como los medios sociales, pero no se ha tenido en cuenta la importancia del análisis de los datos, de gran importancia estratégica por la ingente cantidad de datos que se generan y por la oportunidad que representan para conocer el comportamiento del usuario. Estas habilidades analíticas no sólo afectan a las tareas de marketing; no en vano se ha acuñado el término databrarian, como bibliotecario de datos de cualquier tipo.

Los sitios adaptativos (adaptables al tamaño de la pantalla) serán una constante frente a las apps

Organización. Las empresas deben organizarse de una nueva forma, pues el marketing debe estar presente desde la creación del producto, a lo largo de su desarrollo, la venta, la atención al usuario, etc. Un problema puede ser que todos se consideren responsables de marketing pero nadie lidere. Los líderes deben saber transmitirlo al resto de su empresa porque no sólo ellos sino también el resto de empleados tiene mucho que decir a la hora de hacer marketing y publicidad sobre su compañía y sus productos.

La mayoría de las empresas afirman que están planificando acciones para acometer estos retos. Para el ámbito de las bibliotecas la pregunta sería hasta qué punto somos conscientes de estos retos y de qué forma los estamos afrontando.

Los responsables de bibliotecas deberían centrarse de forma prioritaria en desarrollar habilidades analíticas en su personal y tomar decisiones basadas en datos, para establecer las estrategias de relaciones con sus stakeholders aprovechando la oportunidad que les brindan los medios digitales y sociales para conseguir la verdadera satisfacción de sus usuarios con la marca biblioteca.

\section{Conclusiones}

Los retos que plantea el marketing digital y todos sus frentes obligan a una toma rápida de decisiones no sólo a las empresas sino también a las bibliotecas como organizaciones.

La función del marketing para las bibliotecas va más allá de las campañas ocasionales de promoción de eventos o recursos concretos, online y presenciales. Debe formar parte de un plan estratégico de marketing digital integrado, en el que se hayan segmentado las audiencias, definido los objetivos SMART, establecido los canales apropiados y las acciones tácticas correspondientes y sus sistemas de medición y evaluación. Para ello es imprescindible que las bibliotecas exploten los datos que les ofrecen las plataformas que dan soporte a sus sistemas de gestión y de información, que su personal esté formado en análisis de datos y que a partir de esos datos, de la experiencia del bibliotecario y de la participación directa de los usuarios, se establezcan las estrategias y las tácticas del marketing relacional y de atracción.

Como decía Drucker (1954), los recursos han de destinarse a las oportunidades y no a los problemas. La función de la biblioteca se entenderá únicamente en base a una intercomunicación permanente con sus usuarios que va más allá de conocer sus demandas, condiciones y entorno. Se trata de interesarles para co-crear e innovar en torno a nuestros productos y servicios y la cadena de valor. Los usuarios expresan sus demandas, las bibliotecas se convierten en proveedoras que responden just in time.

El marketing no es una batalla de productos o servicios sino de percepciones y de datos. La gente no compra el mejor producto, sino el que cree que es el mejor, ya que es la emoción del producto lo que realmente vende, no el producto en sí mismo. Sólo aquellas bibliotecas que sean capaces de reinventarse trabajando con sus usuarios alcanzarán el éxito.

\section{Notas}

1. Por intercambio de valor se entienden las transacciones entre el cliente y la empresa, desde dinero por mercancía, hasta información personal por contenidos de interés, engagement por atención personalizada, fidelización por descuentos, etc. Es decir, todos los intercambios que se producen entre cliente y empresa tienen un valor que puede ser económico o de cualquier otro tipo.

2. Post de Robert Rose sobre content marketing forecast http://www.econtentmag.com/Articles/Column/ContentMarketing-Evolution/Content-Marketing-Forecast-100224.htm

3. Post en TicBeat, 5 de noviembre de 2014

http://www.ticbeat.com/tecnologias/estrategia-documentadaelemento-clave-en-marketing-de-contenidos

4. Información procedente de:

http://www.elemprendedor.ec/nuevas-reglas-delmarketing

5. Más información en:

http://blog.newscred.com/article/content-marketing-in2014-cmis-benchmarks-budgets-trends-reports/9429cca7f acdafdb9dc776c76140cb7c 


\section{Entrevista a David Berkowitz}

http://entrevistas.ticbeat.com/entrevista-david-berkowitzdirector-marketing-mry-experto-publicidad

7. Paneles en Pinterest de LibraryAware

http://www.pinterest.com/libraryaware/boards

8. La infografía de Wheelhouse recoge 10 tendencias en marketing online para 2015

http://i.imgur.com/M8p4tlk.png

9. Post de John Greenough

http://www.businessinsider.com/how-the-internet-ofthings-market-will-grow-2014-10

10. Más información en:

http://www.emarketer.com/Article/Smartphones-RuleSpain/1011558/2

11. Informe Social customer service: The future of customer satisfaction.

https://socialbusiness.hootsuite.com/whitepaper-future-ofcustomer-satisfaction.html

12. Noticia en TicBeat, 29 de octubre de 2014

http://www.ticbeat.com/economia/ibm-twitter-alianza

13. Noticia en TicBeat, 9 de noviembre de 2014

http://www.puromarketing.com/30/23099/tendenciasmarketing-digital-nos-depara.html

14. http://dalealaweb.com/2012/01/los-riesgos-en-las-campanasde-redes-sociales-el-caso-mcdonalds

http://www.inc.com/hollis-thomases/mcdonaldsmcdstories-twitter-mess.html

http://www.forbes.com/sites/kashmirhill/2012/01/24/

mcdstories-when-a-hashtag-becomes-a-bashtag

15. Web de los informes Horizon

http://www.nmc.org

16. Big data: The next frontier for competition http://www.mckinsey.com/features/big_data

\section{Bibliografía}

Drucker, Peter F. (1954). The practice of management. New York: Harper Business. ISBN: 0060913169

Elsevier (2014). Marketing the academic library. Elsevier Inc.

Jiménez-Zarco, Ana-Isabel; Martínez-Ruiz, María-Pilar; Llamas-Alonso, María-Rosa (2007). “Creación e intercambio de valor en las relaciones empresa-consumidor". Boletín económico de ICE, Información Comercial Española, n. 2910, pp. 41-60.

Kotler, Philip (1999). El marketing según Kotler: cómo crear, ganar y dominar los mercados. Barcelona: Paidós. ISBN: 9788449324772

Kotler, Philip; Kartajaya, Hermawan; Setiawan, Iwan (2011). Marketing 3.0. Madrid: LID Editorial. ISBN: 9788483568439

Lee, Deborah (2013). "What is marketing?". Public services quaterly, v. 9, n. 2, pp. 169-171.

http://dx.doi.org/10.1080/15228959.2013.785900
Leeflang, Peter S. H.; Verhoef, Peter C.; Dahlström, Peter; Freundt, Tjark (2014). "Challenges and solutions for marketing in a digital era". European management journal, v. 32, n. 1, pp. 1-12.

http://dx.doi.org/10.1016/j.emj.2013.12.001

Martínez-Martínez, Silvia; Lara-Navarra, Pablo (2014). “El big data transforma la interpretación de los medios sociales". El profesional de la información, v. 23, n. 6, pp. 575-581. http://dx.doi.org/10.3145/epi.2014.nov.03

Meerman-Scott, David (2011). The new rules of marketing \& PR. 3르 ed. rev. New Jersey: John Wiley \& Sons.

Merlo-Vega, José-Antonio (2014). "La biblioteca como 'community manager' y 'content curator' (1/2). El ciclo de la gestión de comunidades y contenidos. Biblioblog, 22 de marzo.

http://biblioblog.org/2014/03/22/biblioteca-comunidadescontenidos

Polger, Mark-Aaron; Okamoto, Karen (2013). “Who's spinning the library? Responsibilities of academic librarians who promote article". Library management, v. 34, n. 3, n. 236-253. http://dx.doi.org/10.1108/01435121311310914

Seoane-García, Catuxa (2012). "Reconquistar a usuarios, enamorar a ciudadanos y dialogar con personas: Las Bibliotecas Municipais da Coruña y las redes sociales". En: 6 Congreso nacional de bibliotecas públicas, Burgos.

http://travesia.mcu.es/portalnb/jspui/handle/10421/6623

Serrano-Cobos, Jorge (2014). "Big data y analítica web, estudiar las corrientes y pescar en un océano de datos". El profesional de la información, v. 23, n. 6, pp. 561-566. http://dx.doi.org/10.3145/epi.2014.nov.01

Singh, Rajesh (2009). "Does your library have a marketing culture? Implications for service providers". The journal of academic librarianship, v. 30, n. 3, pp.117-137. http://dx.doi.org/10.1108/01435120910937302

Taylor \& Francis Group (2014). Use of social media by the library current practices and future opportunities. A white paper from Taylor \& Francis. Taylor \& Francis Group.

http://www.tandf.co.uk/journals/access/white-papersocial-media.pdf

Tiago, Maria-Teresa-Pinheiro-Melo-Borges; Veríssimo, José-Manuel-Cristóvão (2014). "Digital marketing and social media: Why bother?". Business horizons, v.57, pp. 703-708. http://dx.doi.org/10.1016/j.bushor.2014.07.002

Valls, Josep-Francesc (2014). Estrategias y tácticas de marketing. Interpretando al consumidor en su kilómetro cero. Barcelona: Bresca Editorial, SL. ISBN: 9788415505235

Villaseca-Morales, David (2014). Innovación y marketing de servicios en la era digital. Madrid: ESIC Business \& Marketing School, 328 pp. ISBN: 9788415986508

Yi, Zhixian; Lodge, Damian; McCausland, Sigrid (2013). "Australian academic librarians' perceptions of marketing services and resources". Library management, v. 34, n. 8, pp. 585-602. http://dx.doi.org/10.1108/LM-01-2013-0002 


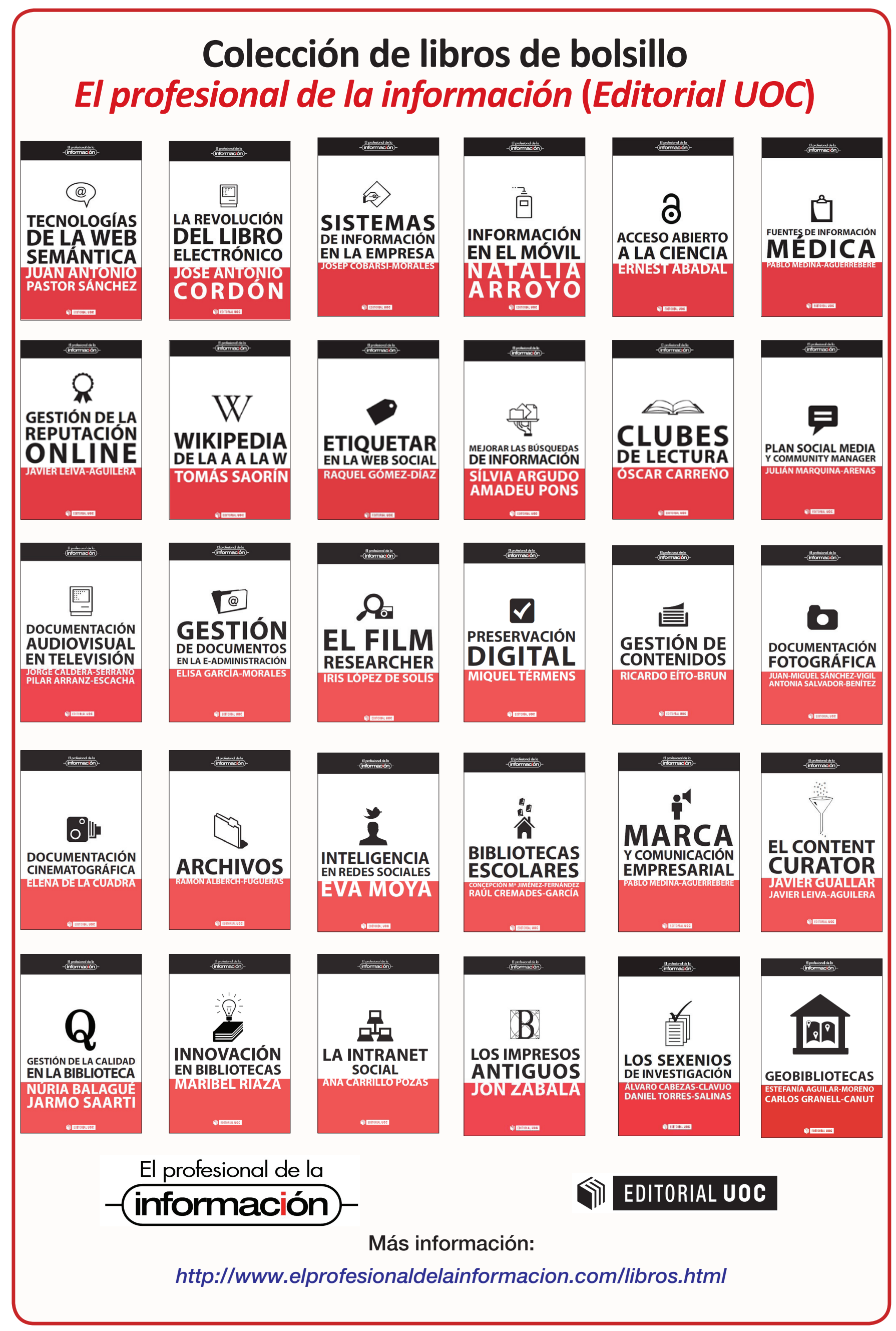

